\title{
Mid-infrared imaging of the massive young star AFGL 2591: Probing the circumstellar environment of an outflow source
}

\author{
Massimo Marengo ${ }^{1,2}$, Ray Jayawardhana ${ }^{1,2,3}$, Giovanni G. Fazio ${ }^{1,2}$, William F. \\ Hoffmann $^{2,4}$, Joseph L. Hora ${ }^{1}$, Aditya Dayal ${ }^{5}$, and Lynne K. Deutsch ${ }^{6}$
}

Received __; accepted _

\footnotetext{
${ }^{1}$ Harvard-Smithsonian Center for Astrophysics, 60 Garden St., Cambridge, MA 02138; Electronic mail: mmarengo@cfa.harvard.edu

${ }^{2}$ Visiting Astronomer, NASA Infrared Telescope Facility

${ }^{3}$ Department of Astronomy, University of California at Berkeley, 601 Campbell Hall, Berkeley, CA 94720; Electronic mail: rayjay@astro.berkeley.edu

${ }^{4}$ Steward Observatory, University of Arizona, Tucson, AZ 85721

${ }^{5}$ IPAC/Caltech, MS 100-22, 770 S. Wilson Ave., Pasadena, CA 91125

${ }^{6}$ Department of Astronomy, CAS 519, Boston University, 725 Commonwealth Ave., Boston, MA 02215
} 


\begin{abstract}
Most, if not all, stars are now believed to produce energetic outflows during their formation. Yet, almost 20 years after the discovery of bipolar outflows from young stars, the origins of this violent phenomenon are not well understood. One of the difficulties of probing the outflow process, particularly in the case of massive embedded stars, is a deficit of high spatial resolution observations. Here, we present sub-arcsecond-resolution mid-infrared images of one massive young stellar object, AFGL 2591, and its immediate surroundings. Our images, at 11.7, 12.5 and $18.0 \mu \mathrm{m}$, reveal a knot of emission $\approx 6 " \mathrm{SW}$ of the star, which may be evidence for a recent ejection event or an embedded companion star. This knot is roughly coincident with a previously seen near-infrared reflection nebula and a radio source, and lies within the known large-scale $\mathrm{CO}$ outflow. We also find a new faint NW source which may be another embedded lower-luminosity star. The IRAS mid-infrared spectrum of AFGL 2591 shows a large silicate absorption feature at $10 \mu \mathrm{m}$, implying that the primary source is surrounded by an optically thick dusty envelope. We discuss the interrelationship of these phenomena and suggest that mid-infrared imaging and spectroscopy provide powerful tools for probing massive star birth.
\end{abstract}

Subject headings: stars:pre-main-sequence-ISM: jets and outflowsstars:individual: AFGL 2591-stars: circumstellar matter 


\section{Introduction}

It is now generally agreed that most, if not all, stars produce energetic outflows during their formation (see Bachiller 1996 for a review). Since the discovery of outflows almost 20 years ago (Snell, Loren \& Plambeck 1980), nearly 200 examples have been found, most of them associated with low-luminosity young stellar objects (YSOs). Outflows can be studied using a variety of tracers including high-velocity CO line emission (Bally \& Lada 1983), shock-excited $\mathrm{H}_{2}$ line emission (e.g., Lane 1989), and optically visible Herbig-Haro (HH) jets (Reipurth 1989, 1999).

All indications are that flows emerge bipolarly from a stellar or circumstellar region. However, the physical processes giving rise to the outflows are not well understood. One suggestion is that a primary wind originating at the star (e.g., Shu et al. 1991) or at the accretion disk (e.g., Pudritz \& Norman 1983) is responsible. In both scenarios, the fast well-collimated wind sweeps up ambient molecular gas in its vicinity, forming two cavities in opposite directions from the star. The displaced molecular gas constitutes the CO outflow and ionized "knots" of gas manifest themselves as HH objects. Outflows probably occur while the star is still accreting material, and may play a significant role in ejecting material of excess angular momentum.

The presence of "bullets" in the CO emission and shocked "knots" visible in the optical and near-infrared suggests that mass ejection from YSOs often occurs in bursts rather than at a steady rate. Such ejection events appear to be intermittent, and the interval between two successive events is on the order of $10^{3}$ years. It is intriguing that the masses and timescales of bullets are similar to those of outbursts observed in FU Ori stars. The FU Ori eruptions are thought to be due to large increases in the accretion rate through a circumstellar disk (Hartmann \& Kenyon 1985). If the two phenomena are related, it may be possible to probe the accretion history of protostars using detailed observations of their 
outflows (e.g., Reipurth 1989).

One of the barriers to better understanding the outflow process is the limited spatial resolution of millimeter-wavelength $\mathrm{CO}$ line observations. The situation is particularly dire in the case of massive young stellar objects in which large extinction $\left(A_{v} \gg 10\right.$ mag $)$ prevents high-resolution optical imaging.

Here we present sub-arcsecond-resolution mid-infrared images of one high-luminosity (Class 0/I) YSO, AFGL 2591, and its immediate surroundings. AFGL 2591 was first recognized as an outflow source by Bally \& Lada (1983). The CO outflow is larger than 1 ' in extent, with the blue lobe extending west or southwest and the red lobe in the northeast direction (Lada et al. 1984; Mitchell, Hasegawa, \& Schella 1992; Hasegawa \& Mitchell 1995). Optical and near-infrared observations have revealed molecular hydrogen emission (40" east and west, Tamura \& Yamashita 1992) and HH objects (10" and 20" west/southwest, Poetzel et al. 1992) near AFGL 2591. The distance to AFGL 2591 is not well established. Assuming a distance of $1 \mathrm{kpc}$, it has a luminosity of $\sim 2 \times 10^{4} L_{\odot}$ and an estimated stellar mass of $10 M_{\odot}$.

\section{Observations and data reduction}

AFGL 2591 was observed on June 3 and 4, 1999 using MIRAC3, the newest version of the University of Arizona/SAO mid-infrared camera (Hoffmann et al. 1998) at the 3.0-meter NASA Infrared Telescope Facility (IRTF). MIRAC3 uses a Boeing HF16 128×128 Si:As blocked impurity band detector. On the IRTF, MIRAC3 has a plate scale of 0.33" / pixel, providing a total field of view of 42 " $\times 42$ ". This pixel scale ensures Nyquist sampling of the diffraction-limited point spread function (PSF).

We obtained images of AFGL 2591 in MIRAC3 11.7, 12.5 and $18.0 \mu \mathrm{m} 10 \%$ passband 
filters, with a total on-source integration time of 600,370 and 540 seconds respectively. The standard stars $\alpha$ Her and $\beta$ Peg, observed before and after the science target, were used for flux and PSF calibration.

We used a standard nodding and chopping technique to remove the background signal, dithering the source on the array to obtain sub-pixel sampling of the PSF. The chop frequency was set to $5 \mathrm{~Hz}$, with a throw of 20 " in the N-S direction. The nod throw was also set to 20" but in the E-W direction, in order to have all four chop-nod beams inside the field of view of the array. Each individual nod cycle required a 10-second on-source integration, and the procedure was repeated for as many cycles as needed to obtain the requested total integration time.

To reduce the data, we have developed our own software written in $\mathrm{C}$ and IDL. We first subtracted the chop-on from the chop-off frames for both nodding beams. The two images thus obtained were then substracted one with the other, in order to get a single frame in which the source appears in all four beams (two negative and two positive). We then applied a gain matrix, derived from images of the dome (high intensity, uniform background) and the sky (low intensity, uniform background), to flat field the chop-nodded image.

This procedure was repeated for each of the nodding cycles for which the source was observed. A final high $\mathrm{S} / \mathrm{N}$ cumulative image was then obtained coadding together all beams, each recentered and shifted on the source centroid. This last coadding was performed on a sub-pixel grid having the size of one-fifth of the original MIRAC3 pixels, thus providing a final pixel scale of $0.066 "$ / pixel. A mask file to block out the effects of bad pixels and field vignetting was also created and applied in this stage, preventing individual rejected pixels from contributing to the final image.

The same observing and reduction procedure was also used for the reference stars, to 
ensure a uniform treatment of the source and the standards.

\section{Results and Discussion}

Figure 1 shows the final MIRAC3 images of AFGL 2591. The central source is not clearly resolved. It has FWHM of $0.84 ", 0.87 "$, and $1.21 "$ at $11.7,12.5$ and $18.0 \mu \mathrm{m}$ respectively. The FWHM of the reference point source $\beta$ Peg in the same filters is 0.82 ", $0.85 "$ and $1.11 "$ respectively. An extended knot of emission $\approx 6 " \mathrm{SW}$ from the central source is visible in all three images. A compact faint source is seen $\approx 11 " \mathrm{NW}$ of AFGL 2591. Figure 2 is a schematic drawing of AFGL 2591, not to scale, with the various observed components indicated.

In Table 1, we list mid-infrared fluxes for the three sources as well as the position angle of SW and NW sources (counter-clockwise from North) and their separation from the central source. The photometry was measured inside apertures of (diameter) 18" (total flux of main and SW sources), 9" (SW source alone) and 5" (NW source). The zero magnitude flux densities for the three filters are 29.2, 25.7 and 12.6 Jy respectively. We estimate an error of $10 \%$ for the photometry, mainly due to the uncertainty of the reference fluxes and the difficulty of isolating the sources when doing aperture photometry. Lower fluxes of the main source were found by Lada et al. (1984) by using broad-band filters at 11.4 and 12.6 $\mu \mathrm{m}$. The discrepancy ( $40 \%$ and $8 \%$ in the two filters) can be explained by the presence of a broad absorption feature in the source spectrum at $10 \mu \mathrm{m}$, which affects broad-band photometry more than our $10 \%$ filters. By fitting the 12.5 and $18.0 \mu \mathrm{m}$ fluxes with a black body, we derive color temperatures of $300-350 \mathrm{~K}$ for the central source, and 100-120K for the SW knot and the NW source.

Persi et al. (1995) presented the only previously published mid-infrared image of 
AFGL 2591. Their observations at $11.7 \mu \mathrm{m}$ were carried out on the 2.1-meter S. Pedro Martir telescope in Baja California, Mexico with an image scale of 1.34" / pixel, and did not reveal the SW knot or the NW compact source seen in our MIRAC3 images, due to the smaller field of view of their detector.

\subsection{Central source}

The flux of the central source rises dramatically from 11.7 to $12.5 \mu \mathrm{m}$, implying that its $11.7 \mu \mathrm{m}$ flux may be suppressed by a large silicate absorption feature at $\sim 10 \mu \mathrm{m}$. Indeed, the low-resolution mid-infrared spectrum obtained by the InfraRed Astronomy Satellite (IRAS) shows deep absorption at that wavelength. Thus, it is likely that the protostar is surrounded by an optically thick dusty envelope, perhaps in free-fall collapse onto the star (van der Tak et al. 1999). Our observations limit the radius of the envelope to $\lesssim 3$ " (or $\lesssim$ $3000 \mathrm{AU}$, assuming a distance of $1 \mathrm{kpc}$ ).

Figure 3 shows the IRAS spectrum of the source, and the best fit we obtain by modeling the circumstellar dust envelope with the radiative transfer code DUSTY (Ivezić et al, 1999). The fit shown is for a spherically symmetric envelope with a radial density profile $\rho \propto r^{-2}$ around a central black body source at effective temperature $T_{\text {eff }}=25,000 \mathrm{~K}$ (van der Tek et al. 1999). We adopted the silicate dust opacity by Ossenkopf et al. (1992), which provides a good fit to the $10 \mu \mathrm{m}$ silicate feature. The fit for $\rho \propto r^{-1.5}$ (i.e., constant infall) is not as good, implying that the infalling envelope is likely to be surrounded by a fair amount of non-infalling cloud material. The model spectrum is not particularly sensitive to the $T_{\text {eff }}$ of the central source. Our best fit to the IRAS spectrum suggests an $A_{V}$ on the order of 100 (derived from the best fit optical depth). 


\subsection{NW source}

AFGL 2591 is generally considered to be an example of relatively isolated massive star formation. However, our detection of a faint point-like source 11" NW of it suggests there may be other embedded low-mass stars in its vicinity. A preliminary look at Two-Micron All-Sky Survey (2MASS) J,H,K images reveals at least two more point-like sources within 40" of AFGL 2591. Deep mid-infrared images of a larger area are needed to search for a possible cluster of low-mass protostars in this region.

\subsection{SW knot}

The SW knot we detect appears close to the inner portion of a loop first observed in the near-infrared by Forrest \& Shure (1986). The near-infrared emission is assumed to arise from scattering of photons from AFGL 2591 off dust grains at the surface of a cavity

cleared by the outflow. The loop, also seen in $\mathrm{NH}_{3}$ observations by Torrelles et al. (1989), is indeed located in the blue outflow lobe, i.e., the one directed toward us. Using Draine \& Lee (1984) opacity at $18 \mu \mathrm{m}$ and assuming $T=100 \mathrm{~K}$ and $d=1 \mathrm{kpc}$, we estimate the mass of this knot to be $>0.2 M_{\odot}$.

The SW knot is also coincident with a radio source as well as a knot of $\mathrm{H}_{2}$ emission. The radio source, detected using interferometers at the Very Large Array (Campbell 1984) and the Owens Valley Radio Observatory (van der Tak et al. 1999), has an approximately flat spectrum between 6.1 and $0.3 \mathrm{~cm}$, and is interpreted as free-free emission from an optically thin HII region. The $\mathrm{H}_{2}$ emission is seen in an image taken with the BEAR imaging spectrometer on the Canada-France-Hawaii telescope using a narrow band filter on the 1-0 S(1) line, after the continuum is subtracted (G. Mitchell, personal communication). The existence of the $\mathrm{H}_{2}$ line implies shock collisional heating of the molecular gas in 
the outflow, which provide the necessary excitation energy $(T \gtrsim 2000 \mathrm{~K}$, see e.g. Smith \& Brand, 1990). On the other hand, the dust responsible for mid-infrared emission is characterized by a much lower local equilibrium temperature of $T_{d} \sim 100 \mathrm{~K}$, as inferred from our photometry. It is likely that the $\mathrm{H}_{2}$ emission comes from the shock itself, while the mid-infrared color temperature is an average through the dust clump.

Previous near-infrared observations have also revealed a second, larger loop of emission at a projected separation of 19" from the star (Burns et al. 1989; Minchin et al. 1991; Tamura et al. 1991; Poetzel, Mundt, \& Ray 1992). The presence of multiple shells/loops is thought to be evidence for episodic mass outflows from AFGL 2591, as are molecular bullets seen in CO emission (Bachiller et al. 1991) and shocked knots observed in the optical and the near-infrared (Reipurth 1989) in other well-known outflow sources. Up to $\sim 1 M_{\odot}$ could be ejected in each eruptive event, though a few tenths of a solar mass is more typical (Bachiller 1996).

If we assume that the SW knot is associated with a recent mass ejection from AFGL 2591, we can estimate its dynamical timescale. Following Minchin et al. (1991), we take that the outflow is inclined from the plane of the sky by 55 degrees, and estimate $\sim 10^{4}$ AU as the true distance from the star to the SW knot. Given a true outflow velocity of 26 $\mathrm{kms}^{-1}$ (based on the line-of-sight velocity of CO gas observed by Bally \& Lada 1983), then we get a dynamical timescale of $\sim 2000$ years. For the same assumptions, the outer loop seen in the near-infrared would have a dynamical timescale of $\sim 6000$ years. Such timescales are consistent with the estimated $10^{4}-10^{5}$-year total duration of the energetic outflow phase of YSOs (Bally \& Lada 1983).

Another possibility is that the SW source itself is an embedded protostar surrounded by an ultracompact HII region. Some Herbig Ae/Be stars - $\mathrm{LkH} \alpha 198$ and $\mathrm{LkH} \alpha$ 234, for example- are known to have heavily embedded mid-infrared companions associated with 
an outflow, a radio source, and HH objects (Lagage et al. 1993; Cabrit et al. 1997). The SW source is well-resolved in our mid-infrared images, with a "coma"-like shape. So, if it is another protostar, its envelope may be affected by the outflow from AFGL 2591.

In summary, our observations suggest that mid-infrared imaging and spectroscopy can be powerful tools for probing the immediate circumstellar environment of embedded stars driving outflows. Such outflows appear to be rather common around young stars and are very likely associated with accretion processes from the early stages of protostars. They may play an important role in determining the mass of the star-disk system, and affect the evolution of the surrounding molecular cloud. Yet our understanding of the outflow process is far from satisfactory. High-resolution imaging with current mid-infrared cameras and future millimeter-wave interferometers could help develop a more unified picture of infall and outflow in star formation.

We are grateful to George Mitchell, Charles Lada and Paolo Persi for helpful discussions and to an anonymous referee for sensible suggestions. We thank the IRTF staff for their outstanding support. The research was supported by the Smithsonian Institution and NASA grants to the SIRTF-IRAC project. 
TABLE 1

MID-INFRARED PHOTOMETRY

\begin{tabular}{lccccc}
\hline \hline Source & $11.7 \mu \mathrm{m}(\mathrm{Jy})$ & $12.5 \mu \mathrm{m}(\mathrm{Jy})$ & $18.0 \mu \mathrm{m}(\mathrm{Jy})$ & PA & Separation \\
\hline \hline $\mathrm{A}$ & $440( \pm 44)$ & $745( \pm 75)$ & $753( \pm 75)$ & - & - \\
$\mathrm{B}$ & $18.4( \pm 2)$ & $15.9( \pm 2)$ & $79.6( \pm 8)$ & $235^{\circ}$ & $6 ”$ \\
$\mathrm{C}$ & $1.8( \pm 0.2)$ & $2.1( \pm 0.2)$ & $16.2( \pm 2)$ & $325^{\circ}$ & $11^{\prime \prime}$ \\
\hline
\end{tabular}




\section{REFERENCES}

Bachiller, R. 1996, ARA\&A, 34, 111

Bachiller, R., Martín-Pintado, J., \& Planesas, P. 1991, A\&A, 251, 639

Bally, J., \& Lada, C.J. 1983, ApJ, 265, 824

Burns, M.S., Hayward, T.L., Thronson, H.A., Jr., \& Johnson, P.E. 1989, AJ, 98, 659

Cabrit, S., Lagage, P.-O., McCaughrean, M., \& Olofsson, G. 1997, A\&A, 321, 523

Campbell, B. 1984, ApJ, 287, 334

Draine, B.T., \& Lee, H.M. 1984, ApJ, 285, 89

Forrest, W.J. \& Shure, M.A. 1986, ApJ, 311, L81

Hartmann, L. \& Kenyon, S.J. 1985, ApJ, 299, 462

Hasegawa, T.I., \& Mitchell, G.F. 1995, ApJ, 451, 225

Hoffmann, W.F., Hora, J.L., Fazio, G.G., Deutsch, L.K., \& Dayal, A. 1998, in Infrared Astronomical Instrumentation, ed. A.M. Fowler, Proc. SPIE 3354, 647

Ivezić, Ž., Nenkova, M., Elitzur, M. 1999, User Manual for DUSTY, University of Kentucky Internal Report, available at http://www.pa.uky.edu/ moshe/dusty

Lada, C.J., Thronson, H.A., Jr., Smith, H.A., Schwartz, P.R., \& Glaccum, W. 1984, ApJ, 286,302

Lagage, P.-O., Olofsson, G., Cabrit, S., Cesarsky, C., Nordh, L., Rodriguez Espinosa, J.M. 1993, ApJ, 417, L79

Lane, A.P. 1989, in Proceedings of the ESO Workshop on Low Mass Star Formation and Pre-main Sequence Objects, ed. Bo Reipurth (Garching bei Munchen: ESO), 331

Minchin, N.R., Hough, J.H., McCall, A., Aspin, C., Hayashi, S.S., Yamashita, T., \& Burton, M.G. 1991, MNRAS, 251, 508 
Mitchell, G.F., Hasegawa, T.I., \& Schella, J. 1992, ApJ, 386, 604

Ossenkopf, V., Henning, Th., Mathis, J.S. 1992, A\&A 261, 567

Persi, P., Ferrari-Toniolo, M., Marenzi, A.R., Busso, M., Corcione, L., Marengo, M., \& Tapia, M. 1995, Ap\&SS, 224, 535

Poetzel, R., Mundt, R., \& Ray, T.P. 1992, A\&A, 262, 229

Pudritz, R.E. \& Norman, C.A. 1983, ApJ, 274, 677

Reipurth, B. 1989, Nature, 340, 42

Reipurth, B. 1999, A General Catalog of Herbig-Haro Objects, available electronically at http://casa.colorado.edu/hhcat

Shu, F.H., Ruden, S.P., Lada, C.J., \& Lizano, S. 1991, ApJ, 370, 31

Smith, M.D., Brand, P.W.J.L. 1990, MNRAS 245, 108

Snell, R.L., Loren, R.B., \& Plambeck, R.L. 1980, ApJ, 239, 17

Tamura, M., Gatley, I., Joyce, R.R., Ueno, M., Suto, H., \& Sekiguchi, M. 1991, ApJ, 378, 611

Tamura, M., \& Yamashita, T. 1992, ApJ, 391, 710

Torrelles, J.M., Ho, P.T.P., Rodríguez, L.F., \& Cantó, J. 1989, ApJ, 343, 222

van der Tak, F.F.S., van Dishoeck, E.F., Evans, N.J., II, Bakker, E.J., \& Blake, G.A. 1999, ApJ, 522, 991 


\section{Figure Captions}

Figure 1. MIRAC3 images of AFGL 2591 at (a) $11.7 \mu \mathrm{m}$, (b) $12.5 \mu \mathrm{m}$, and (c) $18.0 \mu \mathrm{m}$. Contours are plotted for 10 and $15 \sigma$ levels in panels (a) and (b), and for 5, 10 and $15 \sigma$ in panel (c).

Figure 2. A schematic drawing of AFGL 2591 with the various observed components indicated. (Not to scale.)

Figure 3. IRAS mid-infrared spectrum of AFGL 2591 (solid line) and the best-fit model (dashed line) for the $10 \mu \mathrm{m}$ silicate feature. The heavy absorption is presumably due to an optically thick dusty envelope around the massive central star. 

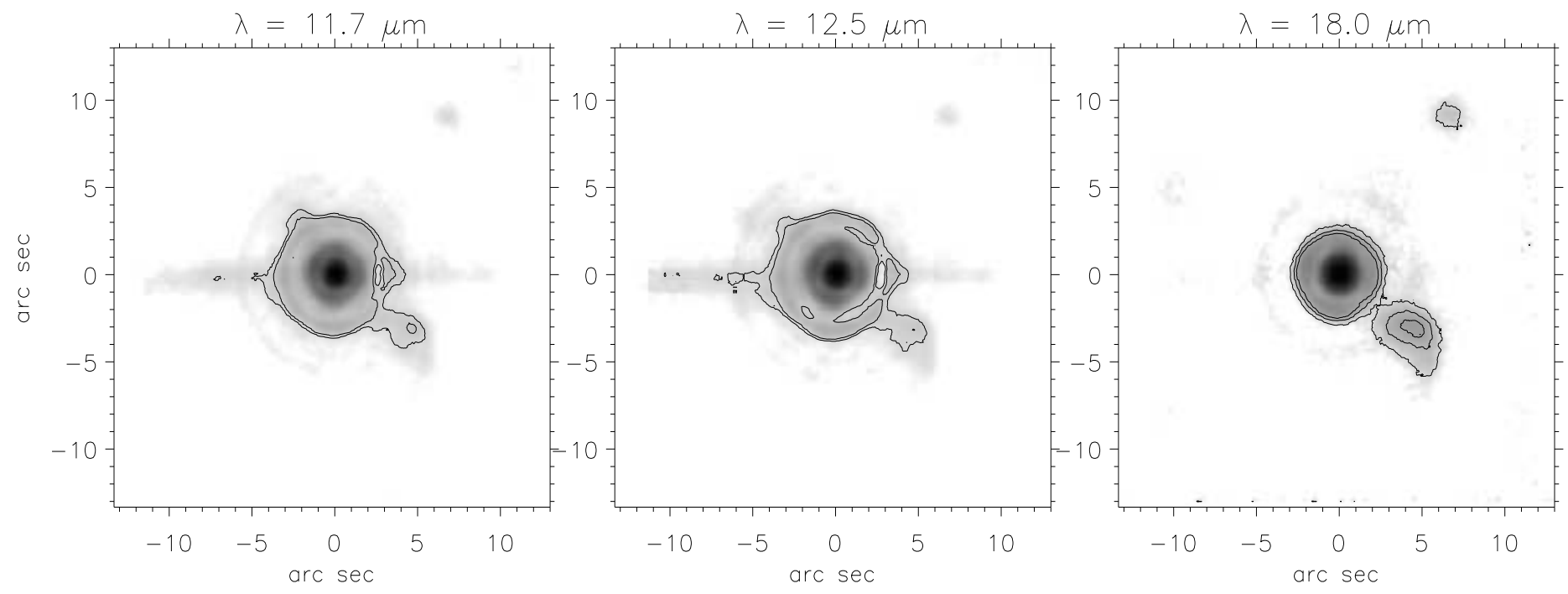

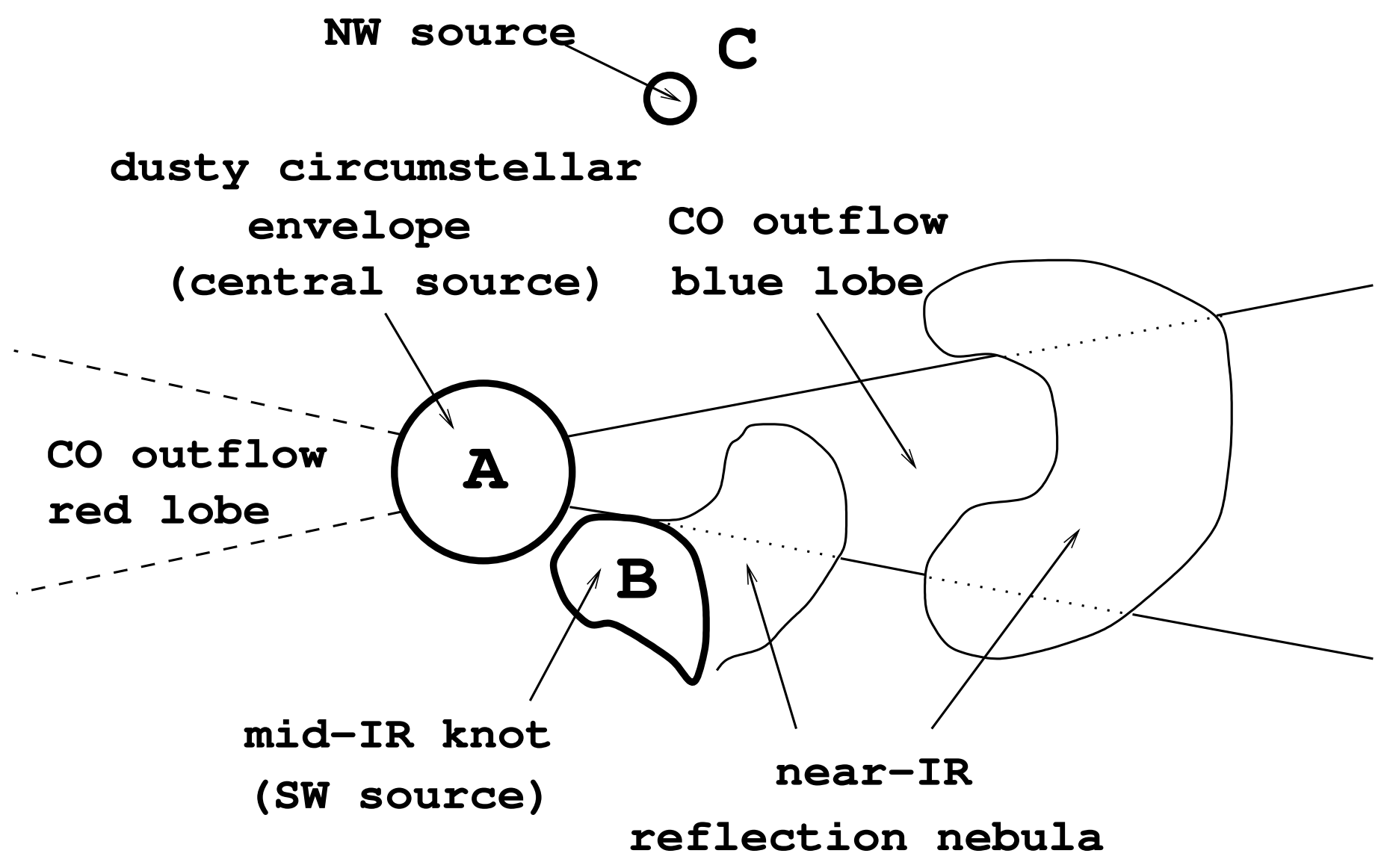


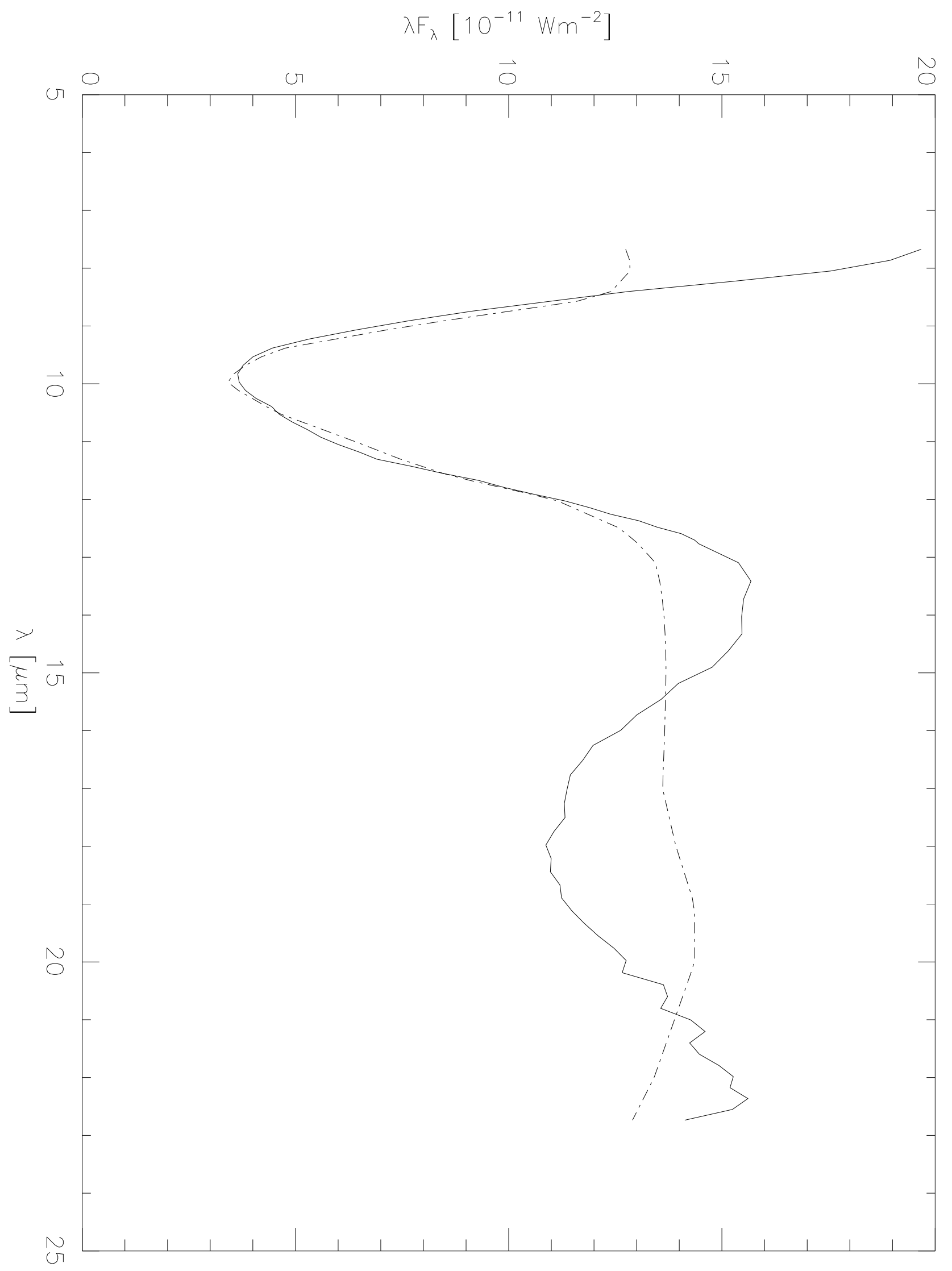

